\title{
Níveis de adubação nitrogenada e bioestimulante na produção e qualidade do algodão BRS verde ${ }^{1}$
}

\author{
Maria M. de Lima ${ }^{2}$, Carlos A. V. de Azevedo ${ }^{3}$, Napoleão E. de M. Beltrão ${ }^{4}$, \\ Vera L. A. de Lima ${ }^{3}$, Maria B. H. do Nascimento ${ }^{3}$ \& Ivana C. de M. Figueirêdo ${ }^{3}$
}

\section{RESUMO}

Objetivou-se, com este trabalho, quantificar os efeitos de adubação nitrogenada e do bioestimulante aplicado nas folhas sobre a produção e qualidade da fibra do algodoeiro colorido BRS Verde. O experimento foi conduzido em condições de casa-de-vegetação do Centro Nacional de Pesquisa do Algodão (Embrapa-CNPA). A adubação nitrogenada foi aplicada em cobertura, nas dosagens de 0,2,9, 5,8 e 8,7 g por planta, equivalentes a 0, 80, 160 e $240 \mathrm{~kg} \mathrm{ha}^{-1}$ de $\mathrm{N}$ e o bioestimulante Stimulate ${ }^{\circledast}$ o foi nas folhas, em dosagens de 0,25 e $0,5 \mathrm{~mL}$ por planta, equivalentes a 250 e $500 \mathrm{~mL} \mathrm{ha}^{-1}$. O delineamento experimental, em blocos ao acaso, tinha nove tratamentos e três repetições em esquema fatorial $4 \times 2$, mais uma testemunha adicional que não recebeu $\mathrm{N}$ nem bioestimulante. Foram avaliadas as variáveis relativas à produção (produção do algodão em caroço, peso de um capulho, número de capulhos, percentagem de fibra e peso de pluma) e às características intrínsecas da fibra (comprimento, uniformidade, índice de fibras, resistência, elongação, finura, reflectância, grau de amarelecimento e fiabilidade). Em relação à variável produção de algodão em caroço por planta, 0 algodoeiro herbáceo respondeu, de maneira linear, a dosagens de $\mathrm{N}$ de até $240 \mathrm{~kg} \mathrm{ha}^{-1}$ (8,7 g por planta). O bioestimulante Stimulate ${ }^{\circledR}$ não alterou a produção nem interagiu com o nitrogênio.

Palavras-chave: algodoeiro herbáceo, características intrínsecas de fibra, manejo de solo

\section{Levels of nitrogen and growth promoter in the production and quality of BRS green cotton}

\begin{abstract}
This research aimed to quantify the effects of nitrogen manuring and growth promoter applied in the leaves on the production and fiber quality of the colored BRS green cotton. The experiment was conducted in greenhouse conditions at the National Center of Cotton Research (Embrapa-CNPA). The nitrogen manuring was applied in the doses of 0, 2.9, 5.8 and 8.7 g per plant, equivalents to $\left(0,80,160\right.$ and $240 \mathrm{~kg} \mathrm{ha}^{-1}$ of N). The Stimulate ${ }^{\circledR}$ growth promoter was applied in the leaves in doses of 0.25 and $0.5 \mathrm{~mL}$ per plant, equivalent to $\left(250\right.$ e $\left.500 \mathrm{~mL} \mathrm{ha}^{-1}\right)$. The experimental design was in randomized blocks with nine treatments and three replications in a factorial scheme of $4 \times 2$, plus an additional control that did not receive nitrogen and growth promoter. The variables related to production (production of cotton, weight of cotton boll, cotton boll number, fiber percentage and feather weight) and to intrinsic characteristics of the fiber (length, uniformity, fiber index, resistance, elongation, fineness, reflectance, yellow degree and spin ability) were analyzed. Considering the cotton production, it was verified that the herbaceous cotton plant responded in a linear way to doses of $\mathrm{N}$ up to $240 \mathrm{~kg} \mathrm{ha}^{-1}$ (8.7 mL per plant). The Stimulate ${ }^{\circledR}$ growth promoter neither alter the production nor interacted with the nitrogen.
\end{abstract}

Key words: herbaceous cotton, intrinsic characteristics of fiber, soil management

1 Artigo extraído da Dissertação de Mestrado do primeiro autor

2 UAEA/CTRN/UFCG. Rua Santo Antônio, 736, Santo Antônio, CEP 58103-355, Campina Grande, PB. Fone: (83) 3362 1586. E-mail: madda-lima@hotmail.com ${ }^{3}$ UAEA/CTRN/UFCG. Av. Aprígio Veloso, 882, Bodocongó, CEP 58109-970, Campina Grande, PB. Fones: (83) 3310 1056; (83) 3310 1349. E-mails: cazevedo@deag.ufcg.edu.br; antunes@deag.ufcg.edu.br

${ }^{4}$ Embrapa Algodão. Rua Osvaldo Cruz, 1143, Centenário, CEP 58107-720, Campina Grande, PB. Fone: (83) 3315 4300. E-mail: nbeltrão@cnpa.embrapa.br 


\section{INTRODUÇÃO}

Quando os portugueses aqui chegaram, o algodão brasileiro já era cultivado, fiado, tecido e usado pelos índios na fabricação de rede e também nas flechas e tochas incendiárias, feitas da pluma. No século XVIII, o algodoeiro se expandiu sobretudo no Pará, Maranhão, Ceará, Pernambuco e Bahia. Com a Guerra da Recessão, em 1865, nos Estados Unidos da América, o algodão brasileiro alcançou enorme desenvolvimento e as exportações do País chegaram a 30,7\% do total produzido (Moraes, 2001).

O algodoeiro herbáceo (Gossypium hirsutum L. raça latifolium Hutch.) é uma das plantas domesticadas mais significativas para a humanidade; sua produção mundial é, hoje, de cerca de 19,16 milhões de toneladas de pluma por ano; além disso, existem em estoque, para consumo, 19,27 milhões de toneladas e produtividade média mundial de $608 \mathrm{~kg}$ de fibra ha-1 (ICAC 2002; CONAB, 2004), com previsão de aumento do consumo mundial em mais de $40 \%$ nos próximos vinte anos (Mariano, 1999).

Conforme Corrêa (1989), o valor do algodoeiro é ressaltado na indústria, onde tudo é aproveitado, especialmente a fibra e a semente; a primeira representa entre 35 a $42 \%$ e a semente, o restante do peso da produção; a fibra é o principal produto do algodão em virtude de apresentar mais de quatrocentas aplicações na indústria, como: confecção de fios para a tecelagem, preparação de algodão hidrófilo, confecção de feltro, cobertores e estofamentos além da obtenção de celulose.

O algodão colorido natural é tão antigo quanto o branco porém só há pouco tempo ele explode comercialmente, após seu melhoramento genético, satisfazendo a demanda de vários segmentos da sociedade de países desenvolvidos, em especial de pessoas que sofrem algum tipo de alergia aos corantes usados no tingimento dos tecidos feitos com o algodão convencional branco (Beltrão, 1999).

Para Carvalho et al. (2002), em 1996 se realizou o cruzamento entre um material introduzido dos EUA, o Arkansas Green, de fibra verde, com a cultivar de fibra branca CNPA 7H, de ampla adaptação à região Nordeste e de fibra de boa qualidade. Dois retrocruzamentos para a cultivar CNPA 7H foram realizados com o objetivo de se recuperar algumas características, especialmente as de fibra deste progenitor.

Ainda conforme Carvalho et al. (2002), a população resultante desses retrocruzamentos foi submetida a seleção genealógica realizando-se, em alguns anos, dois ciclos, com vistas, durante os trabalhos de seleção, à manutenção da cor verde e à resistência da fibra, que outrora era baixa em relação ao doador da cor verde. Obtiveram-se 24 linhagens da cor verde, após vários ciclos de seleção, que chegaram a três linhagens com características superiores em relação às outras, após testes comparativos de rendimento, que compuseram um bulk, do qual a cultivar verde foi originada.

Em 2002, a Embrapa Algodão lançou a cultivar BRS verde, cujas características são: resistência 25,86 gf tex ${ }^{-1}$; comprimento 29,56 (2,5\% mm); rendimento $2.146 \mathrm{~kg} \mathrm{ha}^{-1}$; altura média de plantas $1,27 \mathrm{~m}$; cor da flor e do pólen, creme; um nectário na nervura central; aparecimento do $1^{\circ}$ capulho aos 92 dias; altura do $1^{\circ}$ ramo frutífero $5^{\circ}-6^{\circ}$ nós e ciclo
130 - 140 dias (EMBRAPA, 2002).

Em Azevedo et al. (1998) e Beltrão (1999), a lavoura do algodão exige relativamente grandes quantidades de nitrogênio quando comparado com a demanda por outros elementos, para obtenção do rendimento máximo. Mesmo em se considerando o fato de que a fibra é quase destituída de nitrogênio, as sementes o contêm em grandes quantidades, sendo ele responsável por muitas funções na planta do algodão, que podem afetar seu crescimento e desenvolvimento. O nitrogênio estimula o crescimento e o florescimento, regulariza o ciclo da planta, aumenta a produtividade e melhora o comprimento e a resistência da fibra, quando aplicado em dosagens adequadas.

A quantidade de nitrogênio a ser utilizada na adubação depende das condições climáticas (intensidade e distribuição das chuvas, luminosidade, temperatura etc.), da textura e do teor de matéria orgânica do solo, além do sistema de rotação de culturas adotado. Muitos trabalhos de pesquisa mostram que a resposta de produtividade do algodoeiro à adubação nitrogenada é linear, até a aplicação da dosagem de $120 \mathrm{~kg} \mathrm{ha}^{-1}$ (Grespan \& Zancanaro, 1999).

O nitrogênio é considerado um dos nutrientes mais importantes para o algodoeiro devido a sua interferência nos aspectos qualidade e quantidade da produção; os rendimentos maiores foram relatados em plantas que receberam dosagem de até $150 \mathrm{~kg} \mathrm{ha}^{-1}$ de nitrogênio em cobertura (Lamas \& Staut, 1999; Medeiros et al., 2001).

O uso de biorreguladores e de bioestimulantes vegetais na agricultura, tem mostrado grande potencial no aumento da produtividade de plantas cultivadas. Biorreguladores vegetais são substâncias sintetizadas que, aplicadas exogenamente, possuem ações similares aos grupos de hormônios vegetais conhecidos (citocininas, auxinas, giberelinas, retardadores, inibidores e etileno) (Vieira \& Castro, 2002).

Para Mondino \& Galizzi (2001) quando aplicaram dosagens de $\mathrm{N}$ tanto a 50 quanto a $100 \mathrm{~kg} \mathrm{ha}^{-1}$ melhoraram a resistência da fibra; sobre seu comprimento, a influência foi inconsistente e variou de ano para outro.

Conforme Campos et al. (1995) apud Furlani \& Buzetti. (2001) os quais relataram que, a medida em que se aumentou a dosagem de $\mathrm{N}$ de 0 para 50,100, 150 e $200 \mathrm{~kg} \mathrm{ha}^{-1}$, ocorreu efeito significativo diretamente proporcional, em termos de produtividade do algodão. Grespan \& Zancanaro (1999), Oliveira et al. (1988), Campos et al. (1998) apud Furlani et al. (2001) relataram que a aplicação de dosagens crescentes de $\mathrm{N}$ na cultura do algodoeiro $\left(0,60,120\right.$ e $\left.180 \mathrm{~kg} \mathrm{ha}^{-1}\right)$ promoveu aumento da produtividade para a dosagem de $120 \mathrm{~kg} \mathrm{ha}^{-1}$. Castro et al. (1998), efetuaram pulverização com o fertilizante foliar Microcitros e o bioestimulante "Stimulate ${ }^{\circledR}$ ", em pomar uniforme de laranja pêra (Citrus sinensis L. Osbeck); e constataram incremento no peso médio dos frutos por planta na colheita, em relação ao controle, após a primeira aplicação de Stimulate ${ }^{\circledR}$ de 1,0 L ha-1; já Vieira (2001), quando aplicou 1,6 mL de Stimulate ${ }^{\circledR}$ por $0,5 \mathrm{~kg}$ de sementes obteve uma produção máxima de 16,9 panículas com grãos por planta de arroz.

A cultivar BRS verde foi lançada pela EMBRAPA (2002); como se trata de um novo genótipo, não há, ainda, informações sobre seu comportamento, no tocante a uma 
resposta à adubação nitrogenada e aos promotores de crescimento; objetivou-se, com este trabalho, quantificar os efeitos de adubação nitrogenada e promotor de crescimento aplicado nas folhas sobre a produção e seus componentes do algodoeiro de fibra verde.

\section{MATERIAL E MÉTODOS}

O experimento foi conduzido em condições de casa-devegetação do Centro Nacional de Pesquisa do Algodão (CNPA-EMBRAPA), na cidade de Campina Grande, PB, localizada na zona Centro Oriental do Estado da Paraíba, no Planalto da Borborema, cujas coordenadas geográficas são latitude sul $7^{\circ} 13$ ' 11 ”, longitude oeste $35^{\circ}$ 53' 31” W. Grw. e altitude 547,56 m. O experimento foi instalado em 29/01/ 2003, cuja unidade experimental se compunha de vasos plásticos com aproximadamente $25 \mathrm{~L}$, totalizando vinte e sete vasos, de cor prata fosca, vistas a amenizar os efeitos dos raios solares refletidos por materiais desta cor.

Cada parcela experimental recebeu cerca de $25 \mathrm{~L}$ de solo (coletado da camada superficial 0-30 cm), umedecido até aproximadamente capacidade de campo, com água de abastecimento local; no fundo dos vasos foram feitos dois furos em extremidades opostas, por onde o excesso da água foi drenado.

A profundidade da cova foi de $3 \mathrm{~cm}$ e a adubação de cobertura foi aplicada em um sulco tipo meia lua a $2 \mathrm{~cm}$ de profundidade, enquanto cada parcela experimental recebeu oito sementes plantadas cuja emergência ocorreu 5 dias após a semeadura, com o aparecimento de oito plântulas por vaso; o desbaste foi realizado aos vinte dias após a emergência deixando-se uma plântula por vaso colhendo-se, porém, a de melhor aparência, que foi monitorada durante todo o ciclo fenológico da cultura (140 dias).

Um delineamento experimental em blocos ao acaso com três repetições, em esquema fatorial $4 \times 2$, foi adotado e os fatores foram quatro dosagens de nitrogênio $(0,2,9$, 5,8 e 8,7 g planta $^{-1}$ de $\left.\mathrm{N}\right)$ equivalentes a $\left(0,80,160\right.$ e $\left.240 \mathrm{~kg} \mathrm{ha}^{-1}\right)$, e duas dosagens do promotor de crescimento Stimulate ${ }^{\circledR}(0,25$ e $0,5 \mu \mathrm{L}_{\text {planta }}{ }^{-1}$ ) referentes a (250 e $\left.500 \mu \mathrm{L} \mathrm{ha}^{-1}\right)$, e mais um tratamento adicional ao fatorial (+1), uma testemunha absoluta que não faz parte do fatorial que não recebeu aplicação de $\mathrm{N}$ nem de Stimulate ${ }^{\circledR}$, totalizando 9 tratamentos. O Stimulate ${ }^{\circledR}$ utilizado se constitui dos ácidos indolbutírico (auxina) $0,005 \%$, cinetina (citocinina) 0,009\% giberélico 0,005\%.

Aplicou-se bioestimulante Stimulate ${ }^{\circledR}$ dosagem única, nas folhas, por pulverização aos 26 DAE (dias após a emergência) e se realizaram duas aplicações de nitrogênio em dosagens iguais (a metade da dosagem total), em que a primeira foi realizada na fase de botão floral, aos 34 DAE, e a segunda na fase de floração aos 51 DAE.

O substrato de um solo de textura franco-arenosa utilizado provém do município de Lagoa Seca, PB, classificado como Neossolo Regolítico; coletado nas áreas da Empresa Estadual de Pesquisa Agropecuária (EMEPA) o solo foi caracterizado, química e fisicamente, na Embrapa Algodão e no Laboratório de Irrigação e Salinidade do Departamento de Engenharia Agrícola (UFCG); suas análises químicas e características físico-hídricas estão na Tabela 1.

Incorporou-se composto orgânico na proporção de 5 kg para $100 \mathrm{~kg}$ de solo, que apresentava a seguinte composição química: $\mathrm{pH}=8,6\left(\mathrm{H}_{2} \mathrm{O}\right.$ 1:2.5); $\mathrm{N}=0,56\left(\right.$ dag $\left._{\mathrm{kg}}{ }^{-1}\right) ; \mathrm{k}=1,19$ $\left(\right.$ dag kg-1); $\mathrm{P}=0,58\left(\right.$ dag kg $\left.^{-1}\right) ; \mathrm{Ca}=0,83\left(\right.$ dag kg$\left.^{-1}\right)$; $\mathrm{Mg}=2,22\left(\mathrm{mmol}_{\mathrm{C}} \mathrm{dm}^{-3}\right)$.

Tabela 1. Características químicas e físico-hídricas do solo da área experimental

\begin{tabular}{lcc}
\hline Características & Unidade & Valor \\
$\mathrm{pH}$ em água (1:25) & - & 5,5 \\
$\mathrm{P}$ & $\mathrm{mg} \mathrm{dm}^{-3}$ & 3,81 \\
$\mathrm{~K}$ & $\mathrm{mmol}_{\mathrm{c}} \mathrm{dm}^{-3}$ & 0,10 \\
$\mathrm{Ca}$ & $\mathrm{mmol}_{\mathrm{c}} \mathrm{dm}^{-3}$ & 13,0 \\
$\mathrm{Mg}$ & $\mathrm{mmol}_{\mathrm{c}} \mathrm{dm}^{-3}$ & 6,0 \\
$\mathrm{Al}$ & $\mathrm{mmol}_{\mathrm{c}} \mathrm{dm}^{-3}$ & 1,0 \\
$\mathrm{M} 0$ & $\mathrm{~g} \mathrm{~kg}^{-1}$ & 12,08 \\
Areia & $\mathrm{g} \mathrm{kg}^{-1}$ & 766,5 \\
Silte & $\mathrm{g} \mathrm{kg}^{-1}$ & 86,8 \\
Argila & $\mathrm{g} \mathrm{kg}^{-1}$ & 146,7 \\
Densidade & $\mathrm{kg} \mathrm{m}^{-3}$ & 1,52 \\
\hline
\end{tabular}

O fósforo e o potássio foram aplicados por ocasião do plantio, nas dosagens de 1,3 e 0,73 g planta ${ }^{-1}$ de $\mathrm{P}_{2} \mathrm{O}_{5}$ e $\mathrm{K}_{2} \mathrm{O}$, respectivamente. Utilizaram-se, como fonte para o fósforo e o potássio, o superfosfato triplo $\left(42 \% \mathrm{P}_{2} \mathrm{O}_{5}\right)$ e o cloreto de potássio $\left(62 \% \mathrm{~K}_{2} \mathrm{O}\right)$ e, para o nitrogênio, o sulfato de amônio $(20 \% \mathrm{~N})$.

Por se adequar ao plantio de sequeiro, a variedade BRS Verde foi utilizada em virtude de se adequar ao semi-árido nordestino, devido à baixa incidência de doenças foliares e de solo nesta região. A água usada na irrigação das parcelas experimentais foi a de abastecimento do município de Campina Grande, PB. As irrigações foram realizadas diariamente de forma manual, evitando-se ultrapassar a capacidade de campo.

Avaliaram-se: a) a produção do algodão em caroço e em fibra, os componentes de produção peso de um capulho (valor médio de 20 capulhos), número de capulhos por planta; b) para a fibra, estimaram-se percentagem, peso e as características intrínsecas, comprimento fibrográfico a SL 2,5\%, uniformidade de comprimento, índice de fibras (SFI), resistência (Str), elongação (Elg), finura (micronaire), grau de amarelecimento (+b) e fiabilidade (CSP) do algodão, efetuado pelo Instrumento de Alto Volume (HVI - Higth Volume Instruments), modelo 900, de Spinlab/Zellweger Uster instalado no Laboratório de Fibras e Fios da EMBRAPA/CNPA, Campina Grande, PB, considerando-se a norma internacional ISO 139/ASTM D 1776 /NBR 8428-84 para análise de fibras realizadas em amostras que permaneceram 24 h no Laboratório, em estufa, a uma temperatura de $20 \pm 1^{\circ} \mathrm{C}$ e umidade relativa de $65 \pm 2 \%$, para atingir o equilíbrio higrométrico (aclimatização).

As variáveis da cultura foram avaliadas através da análise de variância, utilizando-se o teste F a nível de 5 e 1\% de probabilidade, conforme Gomes (1985). Para as dosagens de nitrogênio e do promotor de crescimento, análises de regressão 
foram mensuradas, de acordo com Banzatto \& Kronka (1995). O programa utilizado nas análises estatísticas foi o SAS, versão 8.2 (SAS/STAT, 2000).

\section{RESULTADOS E DISCUSSÃO}

Observa-se que a produção, o peso de fibra e o número de capulho por planta, foram significativamente influenciados pela dosagem de nitrogênio aplicado; entretanto, as diferentes dosagens do promotor de crescimento não influenciaram nenhuma dessas variáveis (a produção, o peso de fibra e o número de capulho por planta).

Na Tabela 2 tem-se os resumos das análises de variância para as variáveis referentes à produção e seus componentes. Não houve efeitos significativos para as interações entre os fatores estudados (dosagens de nitrogênio e dosagens do bioestimulante aplicado nas folhas); como se trata de um novo genótipo, não há informações sobre seu comportamento, no tocante a uma resposta aos promotores de crescimento; desta forma, faz-se necessário que novos estudos sejam realizados testando-se dosagens diferentes de Stimulate ${ }^{\circledR}$ tanto em campo como em casa de vegetação.

Tabela 2. Resumo dos quadrados médios das análises de variância dos dados referentes à produção e seus componentes

\begin{tabular}{|c|c|c|c|c|}
\hline Fontes de variação & GL & $\begin{array}{c}\text { Produção } \\
\text { (g por planta) }\end{array}$ & $\begin{array}{c}\text { Peso da fibra } \\
\text { (g) }\end{array}$ & $\begin{array}{l}N^{0} \text { de capulho } \\
\text { por planta (1) }\end{array}$ \\
\hline Nitrogênio & 3 & $1857,11^{* *}$ & $196,71^{\star \star}$ & $1,007 * \star$ \\
\hline Linear & 1 & $2531,1665^{\star *}$ & $107,6819^{n s}$ & $1,1893 * *$ \\
\hline Quadrática & 1 & $202,2534^{*}$ & $67,3327^{\mathrm{ns}}$ & $0,0652^{n s}$ \\
\hline Cúbica & 1 & $52,2387^{\mathrm{ns}}$ & $104,2877^{\mathrm{ns}}$ & $3,3295^{\mathrm{ns}}$ \\
\hline $\begin{array}{l}\text { Promotor de } \\
\text { crescimento }\end{array}$ & 1 & $75,30^{\text {ns }}$ & $12,042^{\mathrm{ns}}$ & $1,05^{\mathrm{ns}}$ \\
\hline NxP & 3 & $170,22^{n s}$ & $22,26^{\mathrm{ns}}$ & $1,23^{\text {ns }}$ \\
\hline $\begin{array}{l}\text { Fatorial Versus } \\
\text { testemunha }\end{array}$ & 1 & $69,69 \mathrm{~ns}$ & $24,67^{\mathrm{ns}}$ & $0,234^{\mathrm{ns}}$ \\
\hline Tratamento & 8 & 778,37 & 86,70 & 0,46 \\
\hline Blocos & 2 & 269,46 & 56,26 & 0,11 \\
\hline Erro & 16 & 241,26 & 29,93 & 0,095 \\
\hline CV\% & - & 27,68 & 32,11 & 11,84 \\
\hline
\end{tabular}

a $1 \%$ pelo teste $F$

Ao contrário de outros trabalhos, como o de Vieira (2001), a aplicação de $1,6 \mathrm{~mL}$ de Stimulate ${ }^{\circledR}$ por $0,5 \mathrm{~kg}$ de sementes levou a uma produção máxima de 16,9 panículas com grãos por planta de arroz, superando, em 13,6\%, a concentração zero de "Stimulate ${ }^{\circledR}$ "; já Castro et al. (1998), efetuaram pulverização com o fertilizante foliar Micro-citros e o bioestimulante “Stimulate ${ }^{\circledR}$ ", em pomar uniforme de laranja pêra (Citrus sinensis L. Osbeck); e notaram aumentos no número de ramos 69 dias após a primeira aplicação de Stimulate ${ }^{\circledR}$ 1,0 L ha-1 além de incremento no peso médio dos frutos por planta na colheita, em relação ao controle.

Para o fator dosagens de nitrogênio, ocorreu significância estatística para as variáveis de produção do algodão em caroço, peso da fibra e número de capulhos por planta; para os contrastes ortogonais testados (Tabela 2) no entanto, não houve significância estatística pelo teste F no nível de probabilidade de 5 e $1 \%$ de probabilidade.

As médias dos tratamentos para as variáveis relacionadas às características intrínsecas da fibra podem ser observadas na Tabela 3. Embora não analisada via ANOVA, devido à falta de algumas parcelas e por não haver amostra da fibra com tamanho suficiente para análise laboratorial, a fibra produzida foi em média de comprimento variado de curta a média, de boa uniformidade, superior a $80 \%$, baixíssimo índice de fibras curtas, menor que 6,0\%, de resistência baixa, estando dentro do padrão da cultivar testada, finura muito baixa, indicando que a ocorrência de aumento da imaturidade devido, possivelmente, ao aumento da temperatura dentro da casa de vegetação, chegou a mais de $50{ }^{\circ} \mathrm{C}$ nos dias de sol; à tarde, por volta das $14 \mathrm{~h}$ e das temperaturas noturnas mais elevadas que o exterior, em virtude da energia geopotencial, e ao efeito estufa no interior da casa de vegetação, também com refletância e grau de amarelecimento dentro do esperado para uma fibra de cor; a fiabilidade foi muito boa, superior a 2000, denotando boa capacidade de fiação da fibra deste tipo de algodão.

Tabela 3. Médias dos tratamentos para as variáveis relacionadas com as características intrínsecas da fibra, em função dos tratamentos testados

\begin{tabular}{|c|c|c|c|c|c|c|c|c|c|}
\hline$T$ & C & U & I & $\mathbf{R}$ & $E$ & $F$ & R & G & $F$ \\
\hline T1 & 27,3 & 84,5 & 5,5 & 28,1 & 7,8 & 4,2 & 75,5 & 10,8 & 2094,5 \\
\hline T2 & 27,5 & 85,2 & 4,8 & 26,7 & 8,1 & 2,9 & 75,6 & 10,7 & 2231,5 \\
\hline T3 & 27,0 & 84,3 & 5,3 & 24,0 & 7,7 & 2,2 & 75,5 & 10,8 & 2238,5 \\
\hline T4 & 27,2 & 85,3 & 4,3 & 27,5 & 7,7 & 3,5 & 75,3 & 10,7 & 2165,8 \\
\hline T5 & 27,4 & 85,0 & 5,0 & 26,3 & 9,2 & 2,9 & 75,5 & 10,6 & 2213,5 \\
\hline T6 & 26,4 & 83,7 & 5,6 & 25,8 & 7,9 & 3,3 & 74,9 & 14,2 & 2119,5 \\
\hline T7 & 27,5 & 84,9 & 4,7 & 27,3 & 7,1 & 3,6 & 75,7 & 10,7 & 2168,3 \\
\hline T8 & 27,3 & 84,4 & 5,3 & 25,3 & 7,4 & 2,6 & 75,8 & 10,8 & 2231,3 \\
\hline T9 & 27,3 & 84,9 & 4,6 & 25,1 & 6,9 & 2,4 & 75,6 & 10,7 & 2250 \\
\hline
\end{tabular}

A melhoria da resistência da fibra obtida nesse estudo (Tabela 3) também pode ser comparada aos dados de Mondino \& Galizzi (2001) que, ao aplicarem dosagens de N, tanto a 50 quanto a $100 \mathrm{~kg} \mathrm{ha}^{-1}$, melhoraram a resistência da fibra; sobre seu comprimento, a influência foi inconsistente e variou de ano para outro.

O nitrogênio aplicado promoveu incremento linear na produção de algodão em caroço (Figura 1A). O número de capulhos por planta também aumentou linearmente com o incremento das dosagens de nitrogênio aplicadas no ambiente edáfico em cobertura (Figura 1B). A produção de algodão em pluma, principal produto desta malvácea, também cresceu com o incremento do nitrogênio ao solo (Figura 1C).

Os resultados apresentados na Figura 1 se equiparam aos obtidos por Medeiros et al. (2001), quanto à produtividade e à altura de planta, que refletem o crescimento e foram alterados pela adição de nitrogênio ao solo correspondendo, os maiores rendimentos, as plantas que receberam até 150 kg N ha-1 em cobertura. 

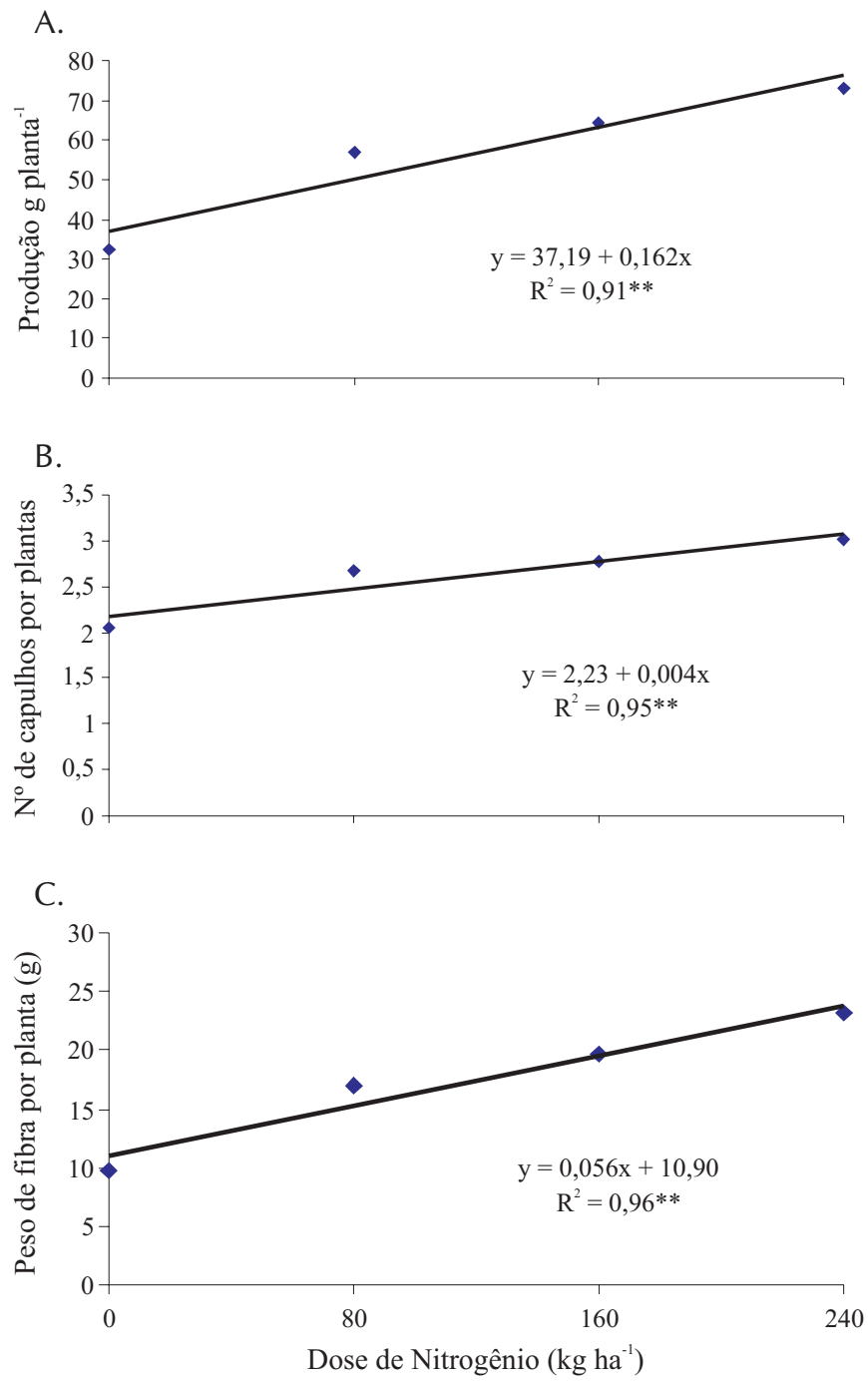

Figura 1. Produção de algodão em caroço (A), número de capulhos por planta (B) e peso de fibra (C) em função das dosagens de nitrogênio

\section{CONCLUSÕES}

1. A cultivar BRS verde apresentou produção de algodão de comportamento linear ascendente em relação a adubação nitrogenada de até $8,7 \mathrm{~g}_{\text {planta-1 }}{ }^{-1}$, equivalente a $240 \mathrm{~kg} \mathrm{ha}^{-1}$.

2. A aplicação foliar de bioestimulante não influiu na produção de algodão nem interagiu com a adubação nitrogenada.

\section{LITERATURA CITADA}

Azevedo, D. M. P. de; Vieira, D. J.; Beltrão, N. E. de M.; Nóbrega, L. B. da. Efeito da adubação nitrogenada e do regulador de crescimento em algodoeiro irrigado. Campina Grande: Embrapa-CNPA, 1998. 4p. Documento, 81.

Banzatto, D. A.; Kronka, S. do N. Experimentação agrícola. 3.ed. Jaboticabal: FUNEP, 1995. 247p.

Beltrão, N. E. de M. Algodão brasileiro em relação ao mundo: situação e perspectivas. In: Beltrão, N. E. de M. O agronegócio do algodão no Brasil. Brasília: Embrapa Comunicação para Transferência de Tecnologia, 1999, v.1,p.15-27.
Carvalho, L. P. de.; Beltrão, N. E. M. de; Costa, J. N. da; Andrade, F. P. de; Silva, O. R. R. F. da; Araújo, G. P. de; Alves, I. BRS Verde. Campina Grande: Embrapa/Algodão PB, 2002. Folder

Castro, P. R. C.; Pacheco, A. C.; Medina, C. L. Efeitos de Stimulate e de micro-citros no desenvolvimento vegetativo e na produtividade da laranjeira pêra. Scientia Agrícola. Piracicaba, v.55, n.2, 7 p, 1998.

CONAB - Companhia Nacional de Abastecimento. Ministério da Agricultura - Pecuária e Abastecimento - MAPA. Brasília, 2004.

Corrêa, J. R. V. Algodoeiro: Informações básicas para seu cultivo. Belém: Embrapa - UEPAE. 1989. 29 p. Documentos, 11

EMBRAPA: Empresa Brasileira de Pesquisa Agropecuária, BRS Verde. Campina Grande: Embrapa Algodão. 2002 folder.

Furlani, E. J.; Buzetti, S. Dosagens e momentos de aplicação de adubo nitrogenado para a cultura do algodoeiro (Gossypium hirsutum L.) IAC 225. In: Congresso Brasileiro do Algodão, 2001, São Paulo. Anais... Campina Grande: Embrapa-CNPA, 2001. 714p

Gomes, F. P. Curso de estatística experimental. 11.ed. Piracicaba: Nobel, 1985, 466p.

Grespan, S. L.; Zancanaro, L. Nutrição e adubação do algodoeiro no Mato Grosso. In: Fundação MT. Mato Grosso: Liderança e Competitividade. Rondonópolis: Fundação MT; Campina Grande: Embrapa - CNPA, 1999. p.87-99.

ICAC. Cotton: review of the world situation. Washington: ICAC. v.55, n.3, 2002, 19p.

Lamas, F. M.; Staut, L. A. Dosagens de nitrogênio e de cloreto de mepiquat no algodoeiro em sistema plantio direto. In: Congresso Brasileiro de Algodão, 2, 1999, Ribeirão Preto. Anais... Campina Grande: Embrapa - CNPA, 1999. 716p.

Mariano, M. Consumo têxtil mundial vai crescer 40\% até 2020. Textília, v.34, p.4-13, 1999.

Medeiros, J. C.; Freire, E. C.; Cunha, H. F.; Queiroz, J. C.; Del' Aqua.; J. M.; Pedroza, M. B.; Assunção, J. H. Safra 1999/ 2000.Principais ações de pesquisa e transferência de tecnologia para o algodoeiro no Estado de Goiás, Campina Grande: Editora: Embrapa-CNPA, 2001 37p.

Mondino, M. H.; Galizzi, F. A. Efects de la aplicación de fertilizantes nitrogenados y fosforados sobre las propriedades tecnologicas de La fibra del algodón producida Bajo Riego. Santiago del Estero Argentina p.1022-1024, 2001.

Moraes, M. V. P. de.; Anuário brasileiro do algodão. Santa Cruz do Sul: Gazeta Santa Cruz, 2001. 143p.

SAS/STAT User's guide. In: SAS Institute. SAS OnlineDoc: version 8.2. Cory, 2000. CD-Rom.

Vieira, E. L.; Ação de bioestimulante na germinação de sementes, vigor de plântulas, crescimento radicular e produtividade de soja (Glycine Max (L.) Merrill), feijoeiro (Phaseolus vulgaris L.) e arroz (Oryza sativa L.). Piracicaba: Dept ${ }^{\circ}$.Ciências Biológicas, USP. 2001. 122p, Tese Doutorado

Vieira, E. L., Castro, P. R. C. Ação de Stimulante no desenvolvimento inicial de plantas de algodoeiro (Gossypium hirsutum L.). Piracicaba: Dept ${ }^{\circ}$. Ciências Biológicas, USP. 2002. 3p.

R. Bras. Eng. Agríc. Ambiental, v.10, n.3, p.619-623, 2006. 